\title{
Zero mode protection at particle-hole symmetry: a geometric interpretation
}

\author{
K. Ziegler \\ Institut für Physik, Universität Augsburg \\ D-86135 Augsburg, Germany
}

(Dated: May 30, 2019)

\begin{abstract}
The properties of zero modes in particle-hole symmetric systems are analyzed in the presence of strong random scattering by a disordered environment. The study is based on the calculation of the time-averaged density distribution on a lattice. In particular, a flat distribution is found for strong random scattering. This result is compared with a decaying distribution for weak random scattering by an analysis of the scattering paths. In the calculation we consider the invariant measure of the average two-particle Green's function, which is related to lattice-covering self-avoiding (LCSA) strings. In particular, strong scattering is associated with LCSA loops, whereas weaker scattering is associated with open LCSA strings. Our results are a generalization of the delocalized state observed at the band center of a one-dimensional tight-binding model with random hopping by Dyson in 1953.
\end{abstract}

\section{INTRODUCTION}

We consider the unitary evolution of a quantum system, which is characterized by the Hamiltonian $H$. Then the time-averaged transition probability $P_{\mathbf{r r}^{\prime}}$ between two quantum states $|\mathbf{r}\rangle \rightarrow\left|\mathbf{r}^{\prime}\right\rangle$ is defined as

$$
P_{\mathbf{r r}^{\prime}}=\lim _{T \rightarrow \infty} \frac{1}{T} \int_{0}^{T}\left|\left\langle\mathbf{r}^{\prime}\left|e^{-i H t}\right| \mathbf{r}\right\rangle\right|^{2} e^{-t / T} d t=\frac{1}{\pi} \lim _{\epsilon \rightarrow 0} \epsilon \int G_{\mathbf{r r}^{\prime}}(E+i \epsilon) G_{\mathbf{r}^{\prime} \mathbf{r}}(E-i \epsilon) d E
$$

with $\epsilon=1 / T$ and the Green's function $G_{\mathbf{r r}^{\prime}}(E+i \epsilon)=\left\langle\mathbf{r}^{\prime}\left|(H-E-i \epsilon)^{-1}\right| \mathbf{r}\right\rangle$. In a fermionic system at low temperature only states near the Fermi surface contribute to transport. Therefore, the main contribution to $P_{\mathbf{r r}^{\prime}}$ comes from the two-particle Green's function

$$
\pi_{\mathbf{r r}^{\prime}}=\frac{1}{\pi} \lim _{\epsilon \rightarrow 0} \epsilon G_{\mathbf{r r}^{\prime}}\left(E_{F}+i \epsilon\right) G_{\mathbf{r}^{\prime} \mathbf{r}}\left(E_{F}-i \epsilon\right),
$$

where $E_{F}=0$ for a particle-hole symmetric system with $H \rightarrow-H$ under particle-hole transformation. On a lattice the state $|\mathbf{r}\rangle$ is a local Wannier state at the site $\mathbf{r}$ and $\pi_{\mathbf{r} \mathbf{r}^{\prime}}$ describes the spatial spreading of the particle density from the source at $\mathbf{r}^{\prime}$ to a site $\mathbf{r}$ which can be considered as a time-averaged density distribution (DD) of the quantum system. Expression (2) can be understood as a classical interpretation of the quantum dynamics in a disordered environment. It has been the foundation of transport in disordered quantum systems, based on the Kubo formalism, and has attracted attention by researchers of different background, ranging from electronic to photonic systems [1-3]. For the latter the time-averaged intensity of a monochromatic electromagnetic field with frequency $\omega$, created by a local source at $\mathbf{r}_{0}=0$, reads [4] [6]

$$
I(\mathbf{r}, \omega)=\lim _{\epsilon \rightarrow 0} \epsilon G_{\mathbf{r} 0}(\omega+i \epsilon) G_{0 \mathbf{r}}(\omega-i \epsilon)\left|j_{0 ; 1}\right|^{2} .
$$

$\left|j_{0 ; 1}\right|^{2}$ is the intensity of the local source.

The connection of the long-range properties of $\left\langle\pi_{\mathbf{r r}}\right\rangle_{d}$, where $\langle\ldots\rangle_{d}$ is the average over a random distribution of scatterers, with symmetries and spontaneous symmetry breaking was discussed early on by Wegner within a functional integral approach 7], who realized that this aspect is related to a nonlinear sigma model [8]. The latter can be derived from the invariant measure in a gradient expansion up to second order. This concept can also be applied to two-dimensional particle-hole symmetric systems. Particle-hole symmetry is important for a large class of physical systems, ranging from superconductors over Dirac fermions to topological materials. In contrast to the general approach of Wegner, the particlehole symmetry implies a reduction of the underlying integration space [9].

Following the standard integration procedure for disordered systems, we must replicate the integration space of both Green's function $(H \pm i \epsilon)^{-1}$ separately, either using a fermion-boson pair or $n$ 
fermion or boson replicas. The reason for replicating both Green's functions separately is that in general $\operatorname{det}(H+i \epsilon) \neq \operatorname{det}(H-i \epsilon)$. On the other hand, in the particle-hole symmetric case with the particle-hole transformation $U H^{T} U^{\dagger}=-H$ ( $U$ is a unitary transformation, ${ }^{T}$ is the matrix transposition), it is sufficient to replicate only $(H+i \epsilon)^{-1}$, since $\operatorname{det}(H-i \epsilon)=\operatorname{det}(-H+i \epsilon)=\operatorname{det}\left(H^{T}+i \epsilon\right)=\operatorname{det}(H+i \epsilon)$. The first equation follows from the fact that $H$ is assumed to be $2 N \times 2 N$ matrix. This enables us to choose, for instance, fermions for $(H+i \epsilon)^{-1}$ and bosons for $\left(H^{T}+i \epsilon\right)^{-1}$. Then it turns out that a rotation in the fermion-bose space is a symmetry transformation that is broken only by the $\epsilon$ term, and the above mentioned spontaneous symmetry breaking is found in the limit $\epsilon \rightarrow 0$. There exists a Grassmann submanifold in the integration space, which is associated with the spontaneously broken symmetry. This is described by an invariant measure, which is the Jacobian for the integration on the the associated submanifold. It depends only on a Grassmann field [10, 11].

Employing the nonlinear sigma model approximation by expanding the invariant measure up to second order in the gradient operator leads to a diffusive behavior in the case of two-dimensional Dirac fermions with diffusion coefficient

$$
D(E)=\lim _{\epsilon \rightarrow 0} \epsilon^{2} \sum_{\mathbf{r}} r_{k}^{2}\left\langle G_{\mathbf{r} 0}(E+i \epsilon) G_{0 \mathbf{r}}(E-i \epsilon)\right\rangle_{d} .
$$

This is not surprising for weak disorder because massless two-dimensional Dirac fermions are diffusive already in the absence of disorder. For stronger disorder this approximation is not sufficient though, as previous calculations have indicated [12]. In other words, the evaluation of the average two-particle Green's function

$$
K_{\bar{r} \bar{r}^{\prime}} \sim\left\langle G_{+; \bar{r} \bar{r}^{\prime}} G_{-; \bar{r}^{\prime} \bar{r}}\right\rangle_{d} \text { with } G_{ \pm}=G(\mp i \epsilon)=(H \pm i \epsilon)^{-1}
$$

must be based on the invariant measure, which neglects exponentially decaying contributions and keeps only the long-range properties. The physics of scattering in $K_{\bar{r} \bar{r}^{\prime}}$ can also be visualized graphically. The individual Green's functions in Eq. (2) can be represented by Feynman path integrals [13 15] for a particle moving from $\mathbf{r}^{\prime}$ to $\mathbf{r}$ and by the complex conjugate integral for a particle moving from $\mathbf{r}$ to $\mathbf{r}^{\prime}$ (reversed path). This pair of paths must be averaged with respect to the disorder distribution to get Eq. (5). The averaging procedure is affected by strong interference, since the Feynman paths are weighted by complex phase factors. As a result of the Grassmann field in the invariant measure, the corresponding paths of $K_{\bar{r} \bar{r}^{\prime}}$ are LCSA strings. Details of these strings, depending on the scattering strength, are discussed in this paper.

The paper is organized as follows. In Sect. [I] the average two-particle Green's function $K_{\bar{r} \bar{r}^{\prime}}$ is represented by the invariant measure and by a Grassmann functional integral with random phases. Then the strong random scattering asymptotics is calculated in Sect. [II], and in Sect. [V] a geometric interpretation of the LCSA strings for strong random scattering (LCSA loops) and weak random scattering (open LCSA strings) are compared. Finally, in Sect. $\mathrm{V}$ the results of the calculation are discussed in a more general context.

\section{AVERAGED TWO-PARTICLE GREEN'S FUNCTION}

Now we consider a spinor Hamiltonian on a lattice of $N$ sites and restrict ourselves to a two-component spinor space. Then the Hamiltonian can be expanded in the Pauli matrix basis as $H=h_{1} \sigma_{1}+h_{2} \sigma_{2}+h_{3} \sigma_{2}$, where the coefficients $h_{j}$ are $N \times N$ matrices on the lattice. The effective average one-particle Green's function at $E=0$ (i.e., at the symmetry point between two bands) reads for the random $2 N \times 2 N$ Hamiltonian $H$ in self-consistent Born approximation

$$
\left\langle(H \pm 2 i \epsilon)^{-1}\right\rangle_{d} \approx g_{ \pm}=\left[H_{0} \pm 2 i(\epsilon+\eta)\right]^{-1}, \quad H_{0}=\langle H\rangle_{d}+\Sigma^{\prime}
$$

where $\Sigma^{\prime}$ is the real part of the self energy and $2 \eta$ is its imaginary part.

The effective Green's function $g_{ \pm}$is the starting point of our study. It has a simple physical interpretation, in which $H_{0}$ describes the propagation of a quantum particle on a lattice and $\eta$ is an effective scattering rate caused by random scattering. $\eta$ is proportional to the density of states at the Fermi level $E_{F}=0[9]$. Thus, it is directly related to the average one-particle Green's function; i.e., it is the 
imaginary part of $g_{-}$. The result can be used as an input to evaluate the average two-particle Green's function (5) as the Grassmann functional integral [16 18]

$$
K_{\bar{r} \bar{r}^{\prime}}=-\frac{1}{\mathcal{N}} \int_{\mathcal{G}} \varphi_{\bar{r}} \varphi_{\bar{r}^{\prime}}^{\prime} J, \quad \mathcal{N}=\int_{\mathcal{G}} J
$$

with the Jacobian $J$ that reads

$$
J^{-1}=\operatorname{det}\left(\mathbf{1}+\varphi \varphi^{\prime}-\varphi h \varphi^{\prime} h^{\dagger}\right), \quad h:=\mathbf{1}-4 i \eta g_{+}, \quad h^{\dagger}:=\mathbf{1}+4 i \eta g_{-} .
$$

Using the definition of $h$ the inverse Jacobian also reads

$$
J^{-1}=\operatorname{det}\left[\mathbf{1}+4 i \eta\left(\varphi g_{+} \varphi^{\prime}-\varphi \varphi^{\prime} g_{-}\right)-16 \eta^{2} \varphi g_{+} \varphi^{\prime} g_{-}\right] .
$$

It was shown in Ref. [12] that the inverse determinant $J$ can be expressed with the help of a random phase $\alpha_{\mathbf{r} j}$ (i.e., with a complex bosonic field) by the replacement $H_{0} \rightarrow \mathbf{H}$ with

$$
\mathbf{H}_{\mathbf{r} j, \mathbf{r}^{\prime} j}=e^{i \alpha_{\mathbf{r} j}} H_{0 ; \mathbf{r} j, \mathbf{r}^{\prime} j^{\prime}} e^{-i \alpha_{\mathbf{r}^{\prime} j^{\prime}}} .
$$

Then the averaged two-particle Green's function becomes a phase averaged Gaussian Grassmann integral

$$
K_{\overline{\mathbf{r}} \overline{\mathbf{r}}^{\prime}}=-\frac{1}{\mathcal{N}}\left\langle\int_{\mathcal{G}} \varphi_{\bar{r}^{\prime}}^{\prime} \varphi_{\bar{r}} \exp \left(\sum_{\mathbf{r}, \mathbf{r}^{\prime}} \varphi_{\mathbf{r}} C_{\mathbf{r r}^{\prime}} \varphi_{\mathbf{r}^{\prime}}^{\prime}\right)\right\rangle_{\alpha}=\frac{\left\langle a d j_{\overline{\mathbf{r}}^{\prime}} C\right\rangle_{\alpha}}{\mathcal{N}},\langle\ldots\rangle_{\alpha}=\frac{1}{2 \pi} \int_{0}^{2 \pi} \ldots \prod_{\mathbf{r}, j} d \alpha_{\mathbf{r} j}
$$

with the adjugate matrix $a d j_{\overline{\mathbf{r}}^{\prime}} C$ and with the normalization factor

$$
\mathcal{N}=\left\langle\int_{\mathcal{G}} \exp \left(\sum_{\mathbf{r}, \mathbf{r}^{\prime}} \varphi_{\mathbf{r}} C_{\mathbf{r r}^{\prime}} \varphi_{\mathbf{r}^{\prime}}^{\prime}\right)\right\rangle_{\alpha}=\langle\operatorname{det} C\rangle_{\alpha}
$$

The $N \times N$ random phase matrix $C$, whose elements are

$$
C_{\mathbf{r} \mathbf{r}^{\prime}}=4 i \eta \sum_{j, j^{\prime}}\left(\mathbf{g}_{\mathbf{r} j, \mathbf{r}^{\prime} j^{\prime}}-\delta_{\mathbf{r} \mathbf{r}^{\prime}} \sum_{\mathbf{r}^{\prime \prime}} \mathbf{g}_{\mathbf{r} j, \mathbf{r}^{\prime \prime} j^{\prime}}^{\dagger}\right)-16 \eta^{2} \sum_{j, j^{\prime}} \mathbf{g}_{\mathbf{r} j, \mathbf{r}^{\prime} j^{\prime}} \sum_{\mathbf{r}^{\prime \prime}, j^{\prime \prime}} \mathbf{g}_{\mathbf{r}^{\prime} j^{\prime}, \mathbf{r}^{\prime \prime} j^{\prime \prime}}^{\dagger}
$$

with $\mathbf{g}=[\mathbf{H}+2 i \bar{\eta}]^{-1}, \bar{\eta}=\eta+\epsilon$, determines the properties of the DD. With $\kappa_{\mathbf{r}}=16 \eta \sum_{\mathbf{r}^{\prime}} \sum_{j, j^{\prime}}\left(\mathbf{g g}^{\dagger}\right)_{\mathbf{r} j, \mathbf{r}^{\prime} j^{\prime}}$ and with the relation $\mathbf{g}-\mathbf{g}^{\dagger}=-4 i \bar{\eta} \mathbf{g g}{ }^{\dagger}$ we get a more compact version of $C$ as

$$
C_{\mathbf{r r}^{\prime}}=\left(\epsilon \kappa_{\mathbf{r}}-\sum_{\mathbf{r}^{\prime \prime}} \Delta_{\mathbf{r r}^{\prime \prime}}\right) \delta_{\mathbf{r r}^{\prime}}+\Delta_{\mathbf{r r}^{\prime}}, \quad \Delta_{\mathbf{r r}^{\prime}}=4 i \eta \sum_{j, j^{\prime}} \mathbf{g}_{\mathbf{r} j, \mathbf{r}^{\prime} j^{\prime}}\left(1+4 i \eta \sum_{\mathbf{r}^{\prime \prime}, j^{\prime \prime}} \mathbf{g}_{\mathbf{r}^{\prime} j^{\prime}, \mathbf{r}^{\prime \prime} j^{\prime \prime}}^{\dagger}\right) .
$$

The form of the matrix $C_{\mathbf{r r}^{\prime}}$ implies $\sum_{\mathbf{r}^{\prime}} C_{\mathbf{r r}^{\prime}}=\epsilon \kappa_{\mathbf{r}}$, such that for $\epsilon=0$ there is a zero eigenvalue with a constant eigenvector, regardless of the specific realization of the random $\mathbf{g}$. Thus, $\operatorname{det} C$ always vanishes in the limit $\epsilon \rightarrow 0$. The very existence of this zero mode is essential for the behavior of the two-particle Green's function. What remains to be discussed is the related propagation for different wavevectors, which is the subject of this paper. For the strong-scattering asymptotics it is easier to start directly from Eq. (91), while for a general discussion in Sect. IV] the random approach of Eqs. (11), (12) is more convenient.

\section{STRONG-SCATTERING ASYMPTOTICS}

Starting from the Jacobian $J$, defined in Eq. (9) on a lattice with $N$ sites, we expand

$$
4 i \eta\left(\varphi g_{+} \varphi^{\prime}-\varphi \varphi^{\prime} g_{-}\right)-16 \eta^{2} \varphi g_{+} \varphi^{\prime} g_{-}
$$


with $\eta / \bar{\eta} \sim 1$ as

$$
4 \frac{\epsilon}{\bar{\eta}} \varphi \varphi^{\prime}-i \frac{1}{\bar{\eta}}\left(\varphi H_{0} \varphi^{\prime}-\varphi \varphi^{\prime} H_{0}\right)+\frac{1}{2 \bar{\eta}^{2}}\left(\varphi H_{0}^{2} \varphi^{\prime}+\varphi \varphi^{\prime} H_{0}^{2}\right)-\frac{1}{\bar{\eta}^{2}} \varphi H_{0} \varphi^{\prime} H_{0}+O\left(\bar{\eta}^{-3}\right),
$$

and rescale the Grassmann field $\varphi \varphi^{\prime} / \bar{\eta} \rightarrow \varphi \varphi^{\prime}$ such that we obtain

$$
-i\left[\varphi H_{0} \varphi^{\prime}-\varphi \varphi^{\prime}\left(H_{0}-4 i \epsilon\right)\right]+\frac{1}{2 \bar{\eta}}\left(\varphi H_{0}^{2} \varphi^{\prime}+\varphi \varphi^{\prime} H_{0}^{2}\right)-\frac{1}{\bar{\eta}} \varphi H_{0} \varphi^{\prime} H_{0}+O\left(\bar{\eta}^{-2}\right) .
$$

Then we get in leading order of $1 / \bar{\eta}$

$$
-\log J=-\left.\sum_{l=1}^{N} \frac{(-1)^{l}}{l} \sum_{\left\{\mathbf{r}_{k}, j_{k}\right\}, 1 \leq k \leq l} A_{\mathbf{r}_{1}, j_{1}, \ldots, \mathbf{r}_{l+1}, j_{l+1}}^{(l)}\right|_{\mathbf{r}_{l+1}=\mathbf{r}_{1}, j_{l+1}=j_{1}},
$$

where

$$
\begin{gathered}
A_{\mathbf{r}_{1}, j_{1}, \ldots, \mathbf{r}_{l+1} j_{l+1}}^{(l)}=i^{l}\left[\varphi_{\mathbf{r}_{1}} \varphi_{\mathbf{r}_{1}}^{\prime} H_{0 ; \mathbf{r}_{1} j_{1}, \mathbf{r}_{2} j_{2}}^{\prime} \cdots \varphi_{\mathbf{r}_{l} j_{l}} \varphi_{\mathbf{r}_{l} j_{l}}^{\prime} H_{0 ; \mathbf{r}_{l} j_{l}, \mathbf{r}_{l+1} j_{l+1}}^{\prime}\right. \\
\left.-\varphi_{\mathbf{r}_{l+1}} \varphi_{\mathbf{r}_{1}}^{\prime} H_{0 ; \mathbf{r}_{1} j_{1}, \mathbf{r}_{2} j_{2}} \cdots \varphi_{\mathbf{r}_{l} j_{l}} \varphi_{\mathbf{r}_{l} j_{l}}^{\prime} H_{0 ; \mathbf{r}_{l} j_{l}, \mathbf{r}_{l+1} j_{l+1}}\right]
\end{gathered}
$$

with $H_{0 ; \mathbf{r} j, \mathbf{r}^{\prime} j^{\prime}}^{\prime}=H_{0 ; \mathbf{r} j, \mathbf{r}^{\prime} j^{\prime}}-4 i \epsilon \delta_{\mathbf{r} \mathbf{r}^{\prime}} \delta_{j j^{\prime}}$. For $\mathbf{r}_{l+1}=\mathbf{r}_{1}$ and $j_{l+1}=j_{1}$ (i.e., for a loop) we obtain

$$
A_{\mathbf{r}_{1}, j_{1}, \ldots, \mathbf{r}_{1} j_{1}}^{(l)}=\left\{\begin{array}{ll}
4 \epsilon \varphi_{\mathbf{r}_{1}} \varphi_{\mathbf{r}_{1}}^{\prime} & l=1 \\
0 & 2 \leq l \leq N
\end{array} .\right.
$$

Thus, the leading order expression results in the simple relation

$$
\operatorname{Tr}\left[\log \left(\mathbf{1}+\varphi \varphi^{\prime}-\varphi h \varphi^{\prime} h^{\dagger}\right)\right]=8 \epsilon \sum_{\mathbf{r}} \varphi_{\mathbf{r}} \varphi_{\mathbf{r}}^{\prime}+O\left(\bar{\eta}^{-1}\right)
$$

for which the Grassmann integration gives

$$
\int_{\mathcal{G}} \exp \left\{-\operatorname{Tr}\left[\log \left(\mathbf{1}+\varphi \varphi^{\prime}-\varphi h \varphi^{\prime} h^{\dagger}\right)\right]\right\} \sim(-8 \epsilon)^{N} .
$$

Next we consider a perturbation of order $1 / \bar{\eta}$. A lengthy but straightforward calculation (App. B) gives for $\epsilon \sim 0$

$$
\int_{\mathcal{G}} \operatorname{det}\left(\mathbf{1}+\varphi \varphi^{\prime}-\varphi h \varphi^{\prime} h^{\dagger}\right)^{-1} \sim(-8 \epsilon)^{N}+\frac{8 \epsilon}{\bar{\eta}} i^{N-2} \int_{\mathcal{G}} \operatorname{Tr}\left[\left(\varphi \varphi^{\prime} H_{0}\right)^{N}\right]+O\left(\bar{\eta}^{-2}\right)
$$

which represents an LCSA loop, and with Eq. (B-

$$
\int_{\mathcal{G}} \varphi_{\overline{\mathbf{r}}} \varphi_{\overline{\mathbf{r}}^{\prime}}^{\prime} \operatorname{det}\left(\mathbf{1}+\varphi \varphi^{\prime}-\varphi h \varphi^{\prime} h^{\dagger}\right)^{-1} \sim-\frac{i^{N-2}}{\bar{\eta}} \sum_{k=0}^{N-2} \int_{\mathcal{G}} \operatorname{Tr}_{2}\left[\left(\varphi \varphi^{\prime} H_{0}\right)_{\overline{\mathbf{r}} \overline{\mathbf{r}}^{\prime}}^{N-1-k}\left(\varphi \varphi^{\prime} H_{0}\right)_{\overline{\mathbf{r}}^{\prime} \overline{\mathbf{r}}}^{k+1}\right]+O\left(\bar{\eta}^{-2}\right)
$$

which represents an LCSA loop with fixed positions $\overline{\mathbf{r}}$ and $\overline{\mathbf{r}}^{\prime}$ with $\overline{\mathbf{r}}^{\prime} \neq \overline{\mathbf{r}}$. For the diagonal case $\overline{\mathbf{r}}^{\prime}=\overline{\mathbf{r}}$ we get

$$
\int_{\mathcal{G}} \varphi_{\overline{\mathbf{r}}} \varphi_{\overline{\mathbf{r}}}^{\prime} \operatorname{det}\left(\mathbf{1}+\varphi \varphi^{\prime}-\varphi h \varphi^{\prime} h^{\dagger}\right)^{-1} \sim(-8 \epsilon)^{N-1}-\frac{i^{N-2}}{\bar{\eta}} \int_{\mathcal{G}} \operatorname{Tr}\left[\left(\varphi \varphi^{\prime} H_{0}\right)^{N}\right]+O\left(\bar{\eta}^{-2}\right) .
$$

For $\overline{\mathbf{r}}^{\prime} \neq \overline{\mathbf{r}}$ the summation over the coordinates $\left\{\mathbf{r}_{k}\right\}(k=1, \ldots, N)$ is actually a (partial) permutation of the lattice sites due to the Grassmann factors $\varphi \varphi^{\prime}$. This implies that the $k$ summation of Eq. (20) is identical to the integral in Eq. (19), except for a factor $1 / N$ :

$$
D:=i^{N-2} \sum_{k=0}^{N-2} \int_{\mathcal{G}} \operatorname{Tr}_{2}\left[\left(\varphi \varphi^{\prime} H_{0}\right)_{\overline{\mathbf{r}}^{\prime} \overline{\mathbf{r}}^{\prime}}^{N-1-k}\left(\varphi \varphi^{\prime} H_{0}\right)_{\overline{\mathbf{r}}^{\prime} \mathbf{\mathbf { r }}}^{k+1}\right]=\frac{i^{N-2}}{N} \int_{\mathcal{G}} \operatorname{Tr}\left[\left(\varphi \varphi^{\prime} H_{0}\right)^{N}\right] .
$$




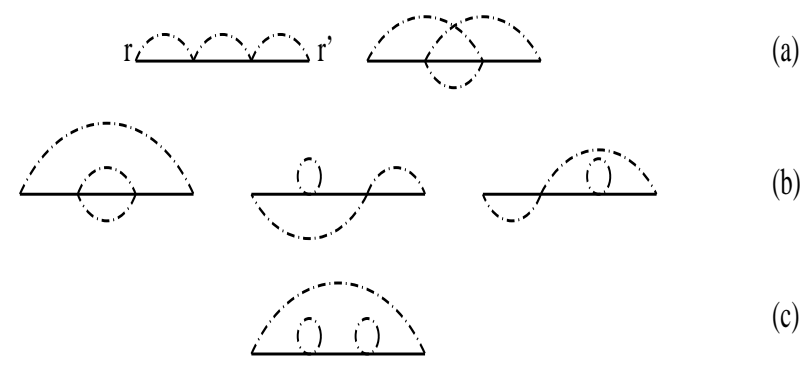

(a)

(b)

FIG. 1: Graphs of $K_{\mathbf{r r}^{\prime}}$ with open LCSA strings after phase averaging for 4 sites. Dashed lines represent $g_{-}$, full lines $g_{+}$. There is one loop in (a), two loops in (b), and three loops in (c).

This follows from the fact that (i) we have for a translational invariant $H_{0}$

$$
\operatorname{Tr}\left[\left(\varphi \varphi^{\prime} H_{0}\right)^{N}\right]=N \operatorname{Tr}_{2}\left[\left(\varphi \varphi^{\prime} H_{0}\right)_{\overline{\mathbf{r}} \mathbf{r}}^{N}\right]
$$

and (ii) the sum over permutations of lattice sites reads for $\overline{\mathbf{r}} \neq \overline{\mathbf{r}}^{\prime}$

$$
\left(\varphi \varphi^{\prime} H_{0}\right)_{\overline{\mathbf{r}} \overline{\mathbf{r}}}^{N}=\sum_{m=1}^{N-1}\left(\varphi \varphi^{\prime} H_{0}\right)_{\overline{\mathbf{r}}^{\prime} \mathbf{r}^{\prime}}^{N-m}\left(\varphi \varphi^{\prime} H_{0}\right)_{\overline{\mathbf{r}}^{\prime} \mathbf{\overline { r }}}^{m}
$$

which gives Eq. (22). Eventually we obtain

$$
K_{\mathbf{r r}^{\prime}}=\frac{\bar{\eta}}{8 \epsilon} \frac{\delta_{\mathbf{r r}^{\prime}}(-8 \epsilon)^{N-1}-\bar{\eta}^{-1} D+O\left(\bar{\eta}^{-2}\right)}{(-8 \epsilon)^{N-1}-N \bar{\eta}^{-1} D+O\left(\bar{\eta}^{-2}\right)},
$$

where the prefactor $\bar{\eta}$ is the result of the rescaling of the Grassmann field. In the limit $\bar{\eta} \rightarrow \infty$ we get strong Anderson localization with vanishing localization length

$$
K_{\mathbf{r r}^{\prime}} \sim \frac{\bar{\eta}}{8 \epsilon} \delta_{\mathbf{r r}^{\prime}}
$$

whereas for a large but finite $\bar{\eta}$ with $\bar{\eta} \ll \epsilon\left(E_{t} / \epsilon\right)^{N}$ ( $E_{t}$ is the tunneling energy of the Hamiltonian $H_{0}$ ) we get

$$
K_{\mathbf{r r}^{\prime}} \sim \frac{\bar{\eta}}{8 N \epsilon} .
$$

This result describes a uniform DD over the entire lattice.

Although $D$ drops out in the first order perturbation, for a deeper insight into the physical origin of the result (26) we must calculate $D$. The spinor trace $T r_{2}$ can be rewritten in terms of phase factors or Ising spins: For the product of $2 \times 2$ matrices $H_{0 ; \mathbf{r}_{k}, \mathbf{r}_{k+1}}$ we replace them by the scalars $\tilde{A}_{\mathbf{r}_{k} \mathbf{r}_{k+1}}=$ $\sum_{j, j^{\prime}} S_{\mathbf{r} j} H_{0 ; \mathbf{r}_{k} j, \mathbf{r}^{\prime} j^{\prime}} S_{\mathbf{r}_{k+1} j^{\prime}}^{*}$, where $S_{\mathbf{r} j}$ is either a phase factor $\exp \left(i \alpha_{\mathbf{r} j}\right)\left(0 \leq \alpha_{\mathbf{r} j}<2 \pi\right)$ [12] or an Ising spin $\left(S_{\mathbf{r} j}= \pm 1\right)$. For simplicity we choose the Ising spin here. Then the trace reads

$$
\operatorname{Tr}_{2}\left(H_{0 ; \mathbf{r}_{1} \mathbf{r}_{2}} \cdots H_{0 ; \mathbf{r}_{n} \mathbf{r}_{1}}\right)=2^{-2 n} \sum_{\left\{S_{\mathbf{r}_{k} j}= \pm 1\right\}}\left(\tilde{A}_{\mathbf{r}_{1} \mathbf{r}_{2}} \cdots \tilde{A}_{\mathbf{r}_{n} \mathbf{r}_{1}}\right)
$$

For the example $H_{0}=h_{1} \sigma_{1}+h_{2} \sigma_{2}$ we obtain

$$
\tilde{A}_{\mathbf{r} \mathbf{r}^{\prime}}=S_{\mathbf{r} 1} S_{\mathbf{r}^{\prime} 2}\left(a_{\mathbf{r r}^{\prime}}+T_{\mathbf{r}} b_{\mathbf{r} \mathbf{r}^{\prime}} T_{\mathbf{r}^{\prime}}\right), \quad T_{\mathbf{r}}=S_{\mathbf{r} 1} S_{\mathbf{r} 2}, \quad a=h_{1}-i h_{2}, \quad b=h_{1}+i h_{2}
$$

and

$$
2^{-2 n} \sum_{\left\{S_{\mathbf{r}_{k} j}= \pm 1\right\}} \tilde{A}_{\mathbf{r}_{1} \mathbf{r}_{2}} \cdots \tilde{A}_{\mathbf{r}_{n} \mathbf{r}_{1}}=a_{\mathbf{r}_{1} \mathbf{r}_{2}} \cdots a_{\mathbf{r}_{n} \mathbf{r}_{1}}+b_{\mathbf{r}_{1} \mathbf{r}_{2}} \cdots b_{\mathbf{r}_{n} \mathbf{r}_{1}}
$$


a)

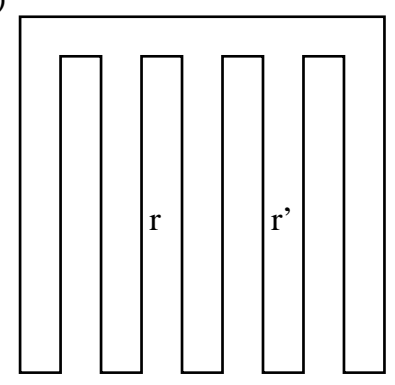

b)

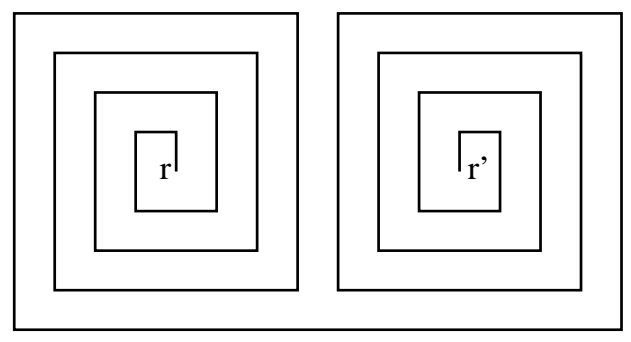

FIG. 2: Examples for LCSA strings: a) a loop from the strong scattering limit of Eq. (20) and b) an open string from the truncated expansion for $a d j_{\mathbf{r r}} \bar{C}$ of Eq. (28) that connects the sites $\mathbf{r}^{\prime}$ and $\mathbf{r}$.

such that we rewrite

$$
D=\frac{1}{N} \sum_{\mathbf{r}_{1}, \ldots, \mathbf{r}_{N}}^{\prime}\left(a_{\mathbf{r}_{1} \mathbf{r}_{2}} \cdots a_{\mathbf{r}_{N} \mathbf{r}_{1}}+b_{\mathbf{r}_{1} \mathbf{r}_{2}} \cdots b_{\mathbf{r}_{N} \mathbf{r}_{1}}\right)
$$

where $\sum_{\mathbf{r}_{1} \ldots, \mathbf{r}_{N}}{ }^{\prime}$ is the restricted sum with $\mathbf{r}_{j} \neq \mathbf{r}_{k}$ for $j \neq k$. Thus, $D$ is represented by the sum of two types of LCSA loops, where the "propagators" are $a=h_{1}-i h_{2}$ and $b=h_{1}+i h_{2}$, respectively.

\section{GEOMETRIC INTERPRETATION}

We have found in Sect. III that the asymptotic behavior of the DD is governed at strong scattering by an LCSA loop (cf. Fig. 2a). On the other hand, for moderate scattering the DD is related to LCSA string with 4-vertices [12]. An example is given for a lattice with four sites in Fig. 1. The 4-vertex graphs resemble the thermal statistics of the two-dimensional Ising model at the critical point [19, 20]. In the following we shall study and compare different contributions to the DD which are characterized according to their geometric structure as LCSA strings. While the actual form and values of the propagators are not relevant, the shape of the LCSA strings is essential. In particular, a single loop vs. an open string that is entangled with other strings belong to different classes (cf. Fig. 2 a and $2 \mathrm{~b}$ ).

In general, an open string from $\mathbf{r}$ to $\mathbf{r}^{\prime}$ on a lattice with $n+2$ sites (cf. Eq. (A1)),

$$
\Delta_{\mathbf{r r}_{1}} \Delta_{\mathbf{r}_{1} \mathbf{r}_{2}} \cdots \Delta_{\mathbf{r}_{n} \mathbf{r}^{\prime}}\left[-\varphi_{\mathbf{r}^{\prime}}^{\prime} \varphi_{\mathbf{r}}+\varphi_{\mathbf{r}}^{\prime} \varphi_{\mathbf{r}}\right] \varphi_{\mathbf{r}_{1}}^{\prime} \varphi_{\mathbf{r}_{1}} \varphi_{\mathbf{r}_{2}}^{\prime} \cdots \varphi_{\mathbf{r}_{n}}
$$

cannot cover the entire lattice with Grassmann variables. This means that we need additional contributions with Grassmann variables. To find those we must distinguish the Grassmann integral of $\mathcal{N}$ in Eq. (12) and the corresponding Grassmann integral for $K_{\overline{\mathbf{r}} \overline{\mathbf{r}}^{\prime}}$ in Eq. (11). Beginning with $\mathcal{N}$, we can only use the diagonal term from the square brackets of Eq. (A1)

$$
\Delta_{\mathbf{r r}_{1}} \Delta_{\mathbf{r}_{1} \mathbf{r}_{2}} \cdots \Delta_{\mathbf{r}_{n} \mathbf{r}^{\prime}} \varphi_{\mathbf{r}}^{\prime} \varphi_{\mathbf{r}} \varphi_{\mathbf{r}_{1}}^{\prime} \varphi_{\mathbf{r}_{1}} \varphi_{\mathbf{r}_{2}}^{\prime} \cdots \varphi_{\mathbf{r}_{n}}
$$

set $\mathbf{r}_{n}=\mathbf{r}$ and borrow a factor $\epsilon \kappa_{\mathbf{r}^{\prime}} \varphi_{\mathbf{r}^{\prime}} \varphi_{\mathbf{r}^{\prime}}^{\prime}$ from the expansion of $\exp \left(-\epsilon \sum_{\mathbf{r}} \kappa_{\mathbf{r}} \varphi_{\mathbf{r}} \varphi_{\mathbf{r}}^{\prime}\right)$. This implies that $\mathcal{N}$ always vanishes with $\epsilon \rightarrow 0$, as already mentioned at the end Sect. II] Geometrically this is an LCSA loop as visualized in Fig. 2a. Moreover, averaging over the random phases of the LCSA string generates additional bridges between lattice sites due to the term $\sum_{\mathbf{r}^{\prime \prime}, j^{\prime \prime}} \mathbf{g}_{\mathbf{r}^{\prime} j^{\prime}, \mathbf{r}^{\prime \prime} j^{\prime \prime}}^{\prime}$ in (14), such that we eventually get a LCSA graph with 4-vertices at each site [12]. These bridges do not occur in the strong scattering limit though, as discussed in the previous section. Thus, the $1 / \bar{\eta}$-expansion is graphically an expansion in terms of 4 -vertices.

In the case of the integral (11) we must take the off-diagonal term from the square brackets in Eq. (A1),

$$
-\Delta_{\mathbf{r r}_{1}} \Delta_{\mathbf{r}_{1} \mathbf{r}_{2}} \cdots \Delta_{\mathbf{r}_{n} \mathbf{r}^{\prime}} \varphi_{\mathbf{r}^{\prime}}^{\prime} \varphi_{\mathbf{r}} \varphi_{\mathbf{r}_{1}}^{\prime} \varphi_{\mathbf{r}_{1}} \varphi_{\mathbf{r}_{2}}^{\prime} \cdots \varphi_{\mathbf{r}_{n}}
$$


and set $\mathbf{r}=\overline{\mathbf{r}}^{\prime}, \mathbf{r}^{\prime}=\overline{\mathbf{r}}$. Thus the extra factor $\varphi_{\bar{r}^{\prime}}^{\prime} \varphi_{\bar{r}}$ in Eq. (11) completes the lattice covering by Grassmann variables. At fixed $\overline{\mathbf{r}}, \overline{\mathbf{r}}^{\prime}$ we get the expression

$$
\left(\varphi_{\overline{\mathbf{r}}^{\prime}} \Delta_{\mathbf{r r}_{1}} \varphi_{\mathbf{r}_{1}}^{\prime}\right)\left(\varphi_{\mathbf{r}_{1}} \Delta_{\mathbf{r}_{1} \mathbf{r}_{2}} \varphi_{\mathbf{r}_{2}}^{\prime}\right) \cdots\left(\varphi_{\mathbf{r}_{n}} \Delta_{\mathbf{r}_{n} \mathbf{r}^{\prime}} \varphi_{\overline{\mathbf{r}}}^{\prime}\right)
$$

which consists of factors of $\varphi_{\mathbf{r}_{k}} \Delta_{\mathbf{r}_{k} \mathbf{r}_{k+1}} \varphi_{\mathbf{r}_{k+1}}^{\prime}$ along a string connecting $\overline{\mathbf{r}}^{\prime}$ and $\overline{\mathbf{r}}$, as depicted in Fig. 20. This string must still be averaged with respect to the random phases. Again, averaging creates bridges between the lattice sites, which results in a graph with 4-vertices [12]. A simple example is depicted in Fig. 1 which has 4 -vertices except for the two external sites $\mathbf{r}$ and $\mathbf{r}^{\prime}$ with 2 -vertices. The full line is the open LCSA string and the dashed lines are the bridges created by phase averaging. To compare this with the strong scattering asymptotic of Sect. III, we can create a loop from an open string with bridges through phase averaging. As a result we get the loop of Fig. 22a. An example with four lattice sites is the graph in Fig. 1(c): after a $1 / \bar{\eta}$ expansion we obtain a loop of the type in Fig. 2h, since both $g_{-}$and $g_{+}$contribute an $H_{0}$ in the leading terms of the expansion.

Phase averaging creates different configurations of bridges between lattice sites. A simple case is one in which the bridges connect neighboring sites along the string, as visualized in left graph of Fig. 1h and in Fig. 3. (This usually does not mean nearest neighbors on the lattice.) This case is formally associated with the replacement $\Delta_{\mathbf{r}_{k} \mathbf{r}_{k+1}}$ by $\left\langle\Delta_{\mathbf{r}_{k} \mathbf{r}_{k+1}}\right\rangle_{\alpha}$ in (28). The same result is obtained from the expression (9) by expanding $-\log J$ up to first order (also known as the nonlinear sigma model approximation of the invariant measure):

$$
-\log J \approx 16 \eta \bar{\eta} \sum_{\mathbf{r}} \operatorname{Tr}_{2}\left(g_{+} g_{-}\right)_{\mathbf{r r}} \varphi_{\mathbf{r}} \varphi_{\mathbf{r}}^{\prime}-16 \eta^{2} \sum_{\mathbf{r}, \mathbf{r}^{\prime}} \operatorname{Tr}_{2}\left(g_{+; \mathbf{r} \mathbf{r}^{\prime}} g_{-; \mathbf{r}^{\prime} \mathbf{r}}\right) \varphi_{\mathbf{r}} \varphi_{\mathbf{r}^{\prime}}^{\prime}
$$

which contains the quadratic form $\sum_{\mathbf{r}, \mathbf{r}^{\prime}} \varphi_{\mathbf{r}} \bar{C}_{\mathbf{r} \mathbf{r}^{\prime}} \varphi_{\mathbf{r}^{\prime}}^{\prime}$ with

$$
\bar{C}_{\mathbf{r r}^{\prime}}=\left\langle C_{\mathbf{r r}^{\prime}}\right\rangle=16 \eta \epsilon r_{2}\left(g_{+} g_{-}\right)_{\mathbf{r r}} \delta_{\mathbf{r r}^{\prime}}+16 \eta^{2}\left[\sum_{\mathbf{r}^{\prime \prime}} \operatorname{Tr}_{2}\left(g_{+; \mathbf{r r}^{\prime \prime}} g_{-; \mathbf{r}^{\prime \prime} \mathbf{r}}\right) \delta_{\mathbf{r r}^{\prime}}-\operatorname{Tr}_{2}\left(g_{+; \mathbf{r} \mathbf{r}^{\prime}} g_{-; \mathbf{r}^{\prime} \mathbf{r}}\right)\right] .
$$

For this example we obtain an approximation of the DD (11) as

$$
K_{\overline{\mathbf{r}} \overline{\mathbf{r}}^{\prime}} \approx \bar{C}_{\overline{\mathbf{r}}^{\prime} \overline{\mathbf{r}}^{\prime}}^{-1}
$$

Thus, the DD decays according to a power law: After a Fourier transformation $\bar{C}_{\mathbf{r}-\mathbf{r}^{\prime}} \rightarrow \tilde{C}_{\mathbf{q}}$ we get $\tilde{C}_{\mathbf{q}} \sim D q^{2}(q \sim 0)$ and

$$
K_{\overline{\mathbf{r}} \overline{\mathbf{r}}^{\prime}} \rightarrow \tilde{K}_{\mathbf{q}}=\frac{1}{\bar{\kappa} \epsilon+D q^{2}+O\left(q^{3}\right)}
$$

with

$$
\bar{\kappa}=16 \eta T r_{2}\left(H_{0}^{2}+4 \bar{\eta}^{2}\right)_{\mathbf{r r}}^{-1}, \quad D=16 \eta^{2} \sum_{\mathbf{r}} r^{2} \operatorname{Tr}_{2}\left(g_{+; \mathbf{r}-\mathbf{r}^{\prime}} g_{-; \mathbf{r}^{\prime}-\mathbf{r}}\right) .
$$

Another example of the random phase representation of the Jacobian in Eqs. (11), (12) uses the meanphase approximation [6, 12]. Since we do not average this leads to a decaying DD as given in Eq. (29) where $\bar{C}_{\mathbf{r r}}$ is replaced by $C_{\mathbf{r r}^{\prime}}$ of Eq. (14) with fixed uniform phases: $e^{i \alpha_{j}}(j=1,2)$, where the two phase parameters $\alpha_{1,2}$ are determined by a variational principle [12]:

$$
H_{0 ; \mathbf{r} j, \mathbf{r}^{\prime} j^{\prime}} \rightarrow \mathcal{H}_{0 ; \mathbf{r} j ; \mathbf{r}^{\prime} j^{\prime}}=H_{0 ; \mathbf{r} j, \mathbf{r}^{\prime} j^{\prime}} e^{i\left(\alpha_{j}-\alpha_{j^{\prime}}\right)} .
$$

The phase factors can also be understood as a rotation when we use a representation in terms of Pauli matrices $H_{0}=h_{1} \sigma_{1}+h_{2} \sigma_{2}+h_{3} \sigma_{3}$ :

$$
\mathcal{H}_{0}=\left(h_{1} c+h_{2} s\right) \sigma_{1}+\left(h_{2} c-h_{1} s\right) \sigma_{2}+h_{3} \sigma_{3}, \quad c=\cos \left(\alpha_{1}-\alpha_{2}\right), \quad s=\sin \left(\alpha_{1}-\alpha_{2}\right)
$$

describes a rotation of $H_{0}$ around the $\sigma_{3}$ axis by the angle $\alpha_{1}-\alpha_{2}$. Since this angle is fixed by a variational principle, the discrete isotropy of the underlying lattice would determine it at discrete values. For $\Delta_{\mathbf{r r}^{\prime}}$ in Eq. (14) this implies

$$
\Delta_{\mathbf{r \mathbf { r } ^ { \prime }}}=-8 i \eta\left[\left(H_{0}^{2}+4 \bar{\eta}^{2}\right)_{j j}^{-1}\left(h_{1} c+h_{2} s\right)\right]_{\mathbf{r} \mathbf{r}^{\prime}}-16 \eta \bar{\eta}\left(H_{0}^{2}+4 \bar{\eta}^{2}\right)_{\mathbf{r} j, \mathbf{r}^{\prime} j}^{-1}
$$




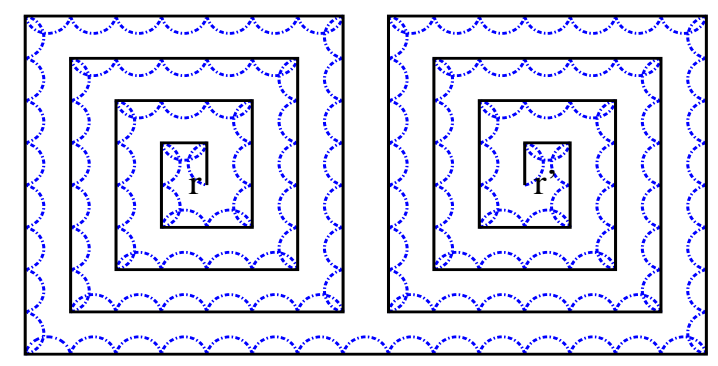

FIG. 3: Example for a loop that consists of an open LCSA string connecting $\mathbf{r}^{\prime}$ and $\mathbf{r}$ which is connected with a sequence of blue dashed bridges. The bridges are created by phase averaging. The links along the LCSA string consist of $g_{+}$propagators and the bridges consist of $g_{-}$propagators.

In the case of two-dimensional Dirac fermions with $h_{1}=q_{1}, h_{2}=q_{2}$ and $h_{3}=m\left(q_{j}\right.$ are the components of the wavevector, $m$ is the Dirac mass) we obtain the result of Ref. [12]:

$$
\tilde{K}_{\mathbf{q}}=\frac{\tau}{4 \epsilon-i \mathbf{s} \cdot \mathbf{q}+2 q^{2} / \tau+O\left(q^{3}\right)}, \quad \tau=\frac{m^{2}+4 \bar{\eta}^{2}}{8 \eta}, \mathbf{s}=(c, s)
$$

The term linear in the wavevector describes a ray mode along the unit vector $\mathbf{s}$, whereas the quadratic term describes the diffusion of the ray mode. For strong scattering $\tau$ is large, which favors linear propagation and suppresses the diffusive behavior. Then the expression $\tilde{K}_{\mathbf{q}}$ has a pole at $\mathbf{s} \cdot \mathbf{q}=-4 i \epsilon$ which is related to the result Eq. (26) in the direction $\mathbf{s}$. An additional summation (or integration) of $\mathbf{s}$ over all directions fixed by the variational principle approximates the constant DD.

\section{DISCUSSION}

Returning to the Feynman paths mentioned in the Introduction, we have found that the contribution of $G_{ \pm}$in Eq. (5) is replaced after averaging with respect to random scattering by an open LCSA string with propagator $g_{+}$from the Grassmann field that is entangled with $g_{-}$strings (bridges) through 4-vertices. Typical examples are depicted in Figs. 10 3. Contributions of the $1 / \bar{\eta}$ expansion to $\left\langle a d j_{\overline{\mathbf{r}}^{\prime}} C\right\rangle_{\alpha}$ in Eq. (24) are discussed in Sect. IV] It is essential to all contributions that there is a zero mode for any realization of the random phase.

Terms of order $1 / \bar{\eta}$ of the $1 / \bar{\eta}$ expansion contribute equally with $H_{0}$ factors from $g_{+}$and $g_{-}$(except for some minus signs), such that we obtain an LCSA loop (Fig. 2h). This implies the uniform DD of Eq. (26). Another additive term to the numerator of expression (24) is the partial sum of Fig. 3 that decays spatially according to a power law in Eq. (30). In this case open LCSA strings must connect the sites $\overline{\mathbf{r}}$ and $\overline{\mathbf{r}}^{\prime}$, which is the reason that $K_{\overline{\mathbf{r}} \overline{\mathbf{r}}^{\prime}}$ decays in space unlike the constant DD in Eq. (26). And finally, an alternative approximation for $\left\langle a d j_{\overline{\mathbf{r}} \overline{\mathbf{r}}^{\prime}} C\right\rangle_{\alpha}$ is a uniform realization of the random phases. It gives ray modes in the direction of the phase difference $\alpha_{1}-\alpha_{2}$ (cf. Eq. (31)).

The main results of Sects. III] IV can be summarized as the "absence of Anderson localization" in the presence of particle-hole symmetry. The two fundamental geometric structures are visualized in Fig. 2 , where Fig. 2a gives a uniform contribution to the numerator of Eq. (26) and Fig. 2b gives a spatially decaying contribution. To obtain these results we must average over a time period $T(=1 / \epsilon)$ which is much longer than the tunneling time $\hbar / E_{t}$ of the Hamiltonian.

Delocalized behavior was observed for one-dimensional systems some time ago, where an extended state was found at the band center of a one-dimensional tight-binding model with random hopping [21 23]. There was also numerical evidence for an extended state at the band center in two-dimensional lattice models 24 27].

The absence of Anderson localization is only valid at the particle-symmetry, which is just a point in the spectrum (e.g., at the Dirac node). We suspect that away from this symmetry point there is a finite localization length, which increases continuously as we approach the symmetry point. This behavior would indicate that in a vicinity of particle-hole symmetric point the localization length is very large. The latter could be a problem in (numerical or real) experiments to detect the Anderson localization length. On 
the other hand, it could be useful for applications to avoid Anderson localization in disordered photonic metamaterials by tayloring the bandstructure with particle-hole symmetry $[28]$.

\section{ACKNOWLEDGMENT}

This work was supported by a grant of the Julian Schwinger Foundation.

\section{Appendix A: Open strings}

The exponential expression in Eqs. (11), (12) can be rewritten with the help of Eq. (14) as

$$
\exp \left(\sum_{\mathbf{r}, \mathbf{r}^{\prime}} \varphi_{\mathbf{r}} C_{\mathbf{r r}^{\prime}} \varphi_{\mathbf{r}^{\prime}}^{\prime}\right)=\exp \left(\epsilon \sum_{\mathbf{r}} \kappa_{\mathbf{r}} \varphi_{\mathbf{r}} \varphi_{\mathbf{r}}^{\prime}\right) \exp \left(\sum_{\mathbf{r}, \mathbf{r}^{\prime}}\left[\sum_{\mathbf{r}^{\prime \prime}} \Delta_{\mathbf{r r}^{\prime \prime}} \delta_{\mathbf{r r}^{\prime}}-\Delta_{\mathbf{r} \mathbf{r}^{\prime}}\right] \varphi_{\mathbf{r}} \varphi_{\mathbf{r}^{\prime}}^{\prime}\right)
$$

with

$$
\kappa_{\mathbf{r}}=16 \eta T r_{2}\left(\mathbf{g g}^{\dagger}\right)_{\mathbf{r r}}, \quad \Delta_{\mathbf{r r}^{\prime}}=16 \eta^{2} \operatorname{Tr}_{2}\left(\mathbf{g}_{\mathbf{r r}^{\prime}} \mathbf{g}_{\mathbf{r}^{\prime} \mathbf{r}}^{\dagger}\right) .
$$

The expansion of the second exponential function leads to two types of open strings on the lattice: type (i) consists of products of $\varphi_{\mathbf{r}} \varphi_{\mathbf{r}}^{\prime} \sum_{\mathbf{r}^{\prime \prime}} \Delta_{\mathbf{r r}^{\prime \prime}}$ links and type (ii) consists of products of $\varphi_{\mathbf{r}} \Delta_{\mathbf{r r}^{\prime}} \varphi_{\mathbf{r}^{\prime}}^{\prime}$ links. Using the relation

$$
\exp \left[\sum_{\mathbf{r}, \mathbf{r}^{\prime}}\left(\sum_{\mathbf{r}^{\prime \prime}} \Delta_{\mathbf{r r}^{\prime \prime}} \delta_{\mathbf{r r}^{\prime}}-\Delta_{\mathbf{r} \mathbf{r}^{\prime}}\right) \varphi_{\mathbf{r}} \varphi_{\mathbf{r}^{\prime}}^{\prime}\right]=\exp \left[\sum_{\mathbf{r}, \mathbf{r}^{\prime}} \varphi_{\mathbf{r}} \Delta_{\mathbf{r} \mathbf{r}^{\prime}}\left(\varphi_{\mathbf{r}}^{\prime}-\varphi_{\mathbf{r}^{\prime}}^{\prime}\right)\right]=\prod_{\left(\mathbf{r}, \mathbf{r}^{\prime}\right)}\left[1-\varphi_{\mathbf{r}} \Delta_{\mathbf{r} \mathbf{r}^{\prime}}\left(\varphi_{\mathbf{r}^{\prime}}^{\prime}-\varphi_{\mathbf{r}}^{\prime}\right)\right]
$$

and assuming that a string has $n+1$ links, we get

$$
\begin{gathered}
{\left[\varphi_{\mathbf{r}} \Delta_{\mathbf{r r}_{1}}\left(\varphi_{\mathbf{r}_{1}}^{\prime}-\varphi_{\mathbf{r}}^{\prime}\right)\right]\left[\varphi_{\mathbf{r}_{1}} \Delta_{\mathbf{r}_{1} \mathbf{r}_{2}}\left(\varphi_{\mathbf{r}_{2}}^{\prime}-\varphi_{\mathbf{r}_{1}}^{\prime}\right)\right] \cdots\left[\varphi_{\mathbf{r}_{n}} \Delta_{\mathbf{r}_{n} \mathbf{r}^{\prime}}\left(\varphi_{\mathbf{r}^{\prime}}^{\prime}-\varphi_{\mathbf{r}_{n}}^{\prime}\right)\right]} \\
=\varphi_{\mathbf{r}} \Delta_{\mathbf{r r}_{1}} \varphi_{\mathbf{r}_{1}}^{\prime} \varphi_{\mathbf{r}_{1}} \Delta_{\mathbf{r}_{1} \mathbf{r}_{2}} \varphi_{\mathbf{r}_{2}}^{\prime} \cdots \varphi_{\mathbf{r}_{n}} \Delta_{\mathbf{r}_{n} \mathbf{r}^{\prime}} \varphi_{\mathbf{r}^{\prime}}^{\prime}+\varphi_{\mathbf{r}} \Delta_{\mathbf{r r}_{1}}\left(-\varphi_{\mathbf{r}}^{\prime}\right) \varphi_{\mathbf{r}_{1}} \Delta_{\mathbf{r}_{1} \mathbf{r}_{2}}\left(-\varphi_{\mathbf{r}_{1}}^{\prime}\right) \cdots \varphi_{\mathbf{r}_{n}} \Delta_{\mathbf{r}_{n} \mathbf{r}^{\prime}}\left(-\varphi_{\mathbf{r}_{n}}^{\prime}\right) \\
=\Delta_{\mathbf{r r}_{1}} \Delta_{\mathbf{r}_{1} \mathbf{r}_{2}} \cdots \Delta_{\mathbf{r}_{n} \mathbf{r}^{\prime}}\left[\varphi_{\mathbf{r}} \varphi_{\mathbf{r}_{1}}^{\prime} \varphi_{\mathbf{r}_{1}} \varphi_{\mathbf{r}_{2}}^{\prime} \cdots \varphi_{\mathbf{r}_{n}} \varphi_{\mathbf{r}^{\prime}}^{\prime}+\varphi_{\mathbf{r}}\left(-\varphi_{\mathbf{r}}^{\prime}\right) \varphi_{\mathbf{r}_{1}}\left(-\varphi_{\mathbf{r}_{1}}^{\prime}\right) \cdots \varphi_{\mathbf{r}_{n}}\left(-\varphi_{\mathbf{r}_{n}}^{\prime}\right)\right] \\
=\Delta_{\mathbf{r r}_{1}} \Delta_{\mathbf{r}_{1} \mathbf{r}_{2}} \cdots \Delta_{\mathbf{r}_{n} \mathbf{r}^{\prime}}\left[\varphi_{\mathbf{r}} \varphi_{\mathbf{r}_{1}}^{\prime} \varphi_{\mathbf{r}_{1}} \varphi_{\mathbf{r}_{2}}^{\prime} \cdots \varphi_{\mathbf{r}_{n}} \varphi_{\mathbf{r}^{\prime}}^{\prime}+\varphi_{\mathbf{r}}^{\prime} \varphi_{\mathbf{r}} \varphi_{\mathbf{r}_{1}}^{\prime} \varphi_{\mathbf{r}_{1}} \cdots \varphi_{\mathbf{r}_{n}}^{\prime} \varphi_{\mathbf{r}_{n}}\right] \\
=\Delta_{\mathbf{r r}_{1}} \Delta_{\mathbf{r}_{1} \mathbf{r}_{2}} \cdots \Delta_{\mathbf{r}_{n} \mathbf{r}^{\prime}}\left[\varphi_{\mathbf{r}}\left(\varphi_{\mathbf{r}_{1}}^{\prime} \varphi_{\mathbf{r}_{1}} \varphi_{\mathbf{r}_{2}}^{\prime} \cdots \varphi_{\mathbf{r}_{n}}\right) \varphi_{\mathbf{r}^{\prime}}^{\prime}+\varphi_{\mathbf{r}}^{\prime} \varphi_{\mathbf{r}}\left(\varphi_{\mathbf{r}_{1}}^{\prime} \varphi_{\mathbf{r}_{1}} \cdots \varphi_{\mathbf{r}_{n}}^{\prime} \varphi_{\mathbf{r}_{n}}\right)\right] \\
=\Delta_{\mathbf{r r}_{1}} \Delta_{\mathbf{r}_{1} \mathbf{r}_{2}} \cdots \Delta_{\mathbf{r}_{n} \mathbf{r}^{\prime}}\left[-\varphi_{\mathbf{r}^{\prime}}^{\prime} \varphi_{\mathbf{r}}+\varphi_{\mathbf{r}}^{\prime} \varphi_{\mathbf{r}}\right] \varphi_{\mathbf{r}_{1}}^{\prime} \varphi_{\mathbf{r}_{1}} \varphi_{\mathbf{r}_{2}}^{\prime} \cdots \varphi_{\mathbf{r}_{n}}
\end{gathered}
$$

This expression vanishes for $\mathbf{r}^{\prime}=\mathbf{r}$ (i.e., for a loop).

\section{Appendix B: Strong-scattering Expansion}

From the expression (9)

$$
\operatorname{Tr} \log \left[\mathbf{1}+4 i \eta\left(\varphi g_{+} \varphi^{\prime}-\varphi \varphi^{\prime} g_{-}\right)-16 \eta^{2} \varphi g_{+} \varphi^{\prime} g_{-}\right]
$$

we get with (15) after a rescaling $\varphi \varphi^{\prime} / \bar{\eta} \rightarrow \varphi \varphi^{\prime}$

$\operatorname{Tr} \log \left[\mathbf{1}+\varphi \varphi^{\prime} A^{\prime}-\varphi A \varphi^{\prime}\right]+\frac{1}{\bar{\eta}} \operatorname{Tr}\left[\left(\mathbf{1}+\varphi \varphi^{\prime} A^{\prime}-\varphi A \varphi^{\prime}\right)^{-1}\left(-\varphi H_{0} \varphi^{\prime} H_{0}+\frac{1}{2} \varphi \varphi^{\prime} H_{0}^{2}+\frac{1}{2} \varphi H_{0}^{2} \varphi^{\prime}\right)\right]+O\left(\bar{\eta}^{-2}\right)$, 
where $A=i H_{0}, A^{\prime}=i H_{0}+4 \epsilon$. The first term on the right-hand side gives with Eq. (17)

$$
\operatorname{Tr} \log \left[\mathbf{1}+\varphi \varphi^{\prime} A^{\prime}-\varphi A \varphi^{\prime}\right]=8 \epsilon \sum_{\mathbf{r}} \varphi_{\mathbf{r}} \varphi_{\mathbf{r}}^{\prime}
$$

such that (B1) reads

$$
8 \epsilon \sum_{\mathbf{r}} \varphi_{\mathbf{r}} \varphi_{\mathbf{r}}^{\prime}+\frac{1}{\bar{\eta}} \sum_{l=0}^{N-1}\left\{\operatorname{Tr}\left[\left(\varphi \varphi^{\prime} A^{\prime}\right)^{l} \varphi \varphi^{\prime} H_{0}^{2}\right]-\sum_{k=0}^{l} \operatorname{Tr}\left[\left(\varphi \varphi^{\prime} A^{\prime}\right)^{l-k}\left(-\varphi A \varphi^{\prime}\right)^{k} \varphi H_{0} \varphi^{\prime} H_{0}\right]\right\}+O\left(\bar{\eta}^{-2}\right) .
$$

For the expressions in Eq. (7) we need

$$
\exp \left\{-\operatorname{Tr} \log \left[\mathbf{1}+4 i \eta\left(\varphi g_{+} \varphi^{\prime}-\varphi \varphi^{\prime} g_{-}\right)-16 \eta^{2} \varphi g_{+} \varphi^{\prime} g_{-}\right]\right\}
$$

which reads with the above expansion

$\exp \left[-8 \epsilon \sum_{\mathbf{r}} \varphi_{\mathbf{r}} \varphi_{\mathbf{r}}^{\prime}\right]\left[1-\frac{1}{\bar{\eta}} \sum_{l=0}^{N-1}\left\{\operatorname{Tr}\left[\left(\varphi \varphi^{\prime} A^{\prime}\right)^{l} \varphi \varphi^{\prime} H_{0}^{2}\right]-\sum_{k=0}^{l} \operatorname{Tr}\left[\left(\varphi \varphi^{\prime} A^{\prime}\right)^{l-k}\left(-\varphi A \varphi^{\prime}\right)^{k} \varphi H_{0} \varphi^{\prime} H_{0}\right]\right\}+O\left(\bar{\eta}^{-2}\right)\right]$.

It should be noticed that the diagonal term of $A^{\prime}$ does not contribute due to the Grassmann factors. Therefore, we can replace $A^{\prime} \rightarrow A$ here. Since the Grassmann integation requires a lattice covering of the Grassmann field we need only the highest number of $l$ in the sum over $l$. The contribution for $l=N-1$

$$
\operatorname{Tr}\left[\left(\varphi \varphi^{\prime} A\right)^{N-1} \varphi \varphi^{\prime} H_{0}^{2}\right]-\sum_{k=0}^{N-1} \operatorname{Tr}\left[\left(\varphi \varphi^{\prime} A\right)^{N-1-k}\left(-\varphi A \varphi^{\prime}\right)^{k} \varphi H_{0} \varphi^{\prime} H_{0}\right]=0
$$

vanishes, as it can be seen by direct inspection of the sum:

$$
\operatorname{Tr}\left[\left(\varphi \varphi^{\prime} A\right)^{N-1} \varphi \varphi^{\prime} H_{0}^{2}\right]=i^{N-1} \operatorname{Tr}\left[\left(H_{0} \varphi \varphi^{\prime}\right)^{N} H_{0}\right]
$$

and the second term simplifies to same expression, since the Grassmann variables in the product must have $N$ different coordinates. The next term with $l=N-2$ in the sum gives a non-vanishing expression

$$
\operatorname{Tr}\left[\left(\varphi \varphi^{\prime} A\right)^{N-2} \varphi \varphi^{\prime} H_{0}^{2}\right]-\sum_{k=0}^{N-2} \operatorname{Tr}\left[\left(\varphi \varphi^{\prime} A\right)^{N-2-k}\left(-\varphi A \varphi^{\prime}\right)^{k} \varphi H_{0} \varphi^{\prime} H_{0}\right] .
$$

The second term also reads

$$
\begin{gathered}
i^{N-2} \operatorname{Tr}\left[H_{0}\left(\varphi \varphi^{\prime} H_{0}\right)^{N-2-k} \varphi\left(-H_{0} \varphi^{\prime} \varphi\right)^{k} H_{0} \varphi^{\prime}\right] \\
=i^{N-2} \sum_{\mathbf{r} \mathbf{r}^{\prime}} \operatorname{Tr}_{2}\left\{\left[H_{0}\left(\varphi \varphi^{\prime} H_{0}\right)^{N-2-k}\right]_{\mathbf{r}^{\prime} \mathbf{r}} \varphi_{\mathbf{r}}\left[H_{0}\left(\varphi \varphi^{\prime} H_{0}\right)^{k}\right]_{\mathbf{r} \mathbf{r}^{\prime}} \varphi_{\mathbf{r}^{\prime}}^{\prime}\right\}
\end{gathered}
$$

This expression survives the Grassmann integration of Eq. (B2) only if $\mathbf{r}^{\prime}=\mathbf{r}$. Thus, we can write for Eq. (B3)

$$
i^{N-2}\left\{\operatorname{Tr}\left[H_{0}\left(\varphi \varphi^{\prime} H_{0}\right)^{N-1}\right]-\sum_{k=0}^{N-1} \sum_{\mathbf{r}} \operatorname{Tr}_{2}\left(\left[H_{0}\left(\varphi \varphi^{\prime} H_{0}\right)^{N-2-k}\right]_{\mathbf{r r}}\left[\left(\varphi \varphi^{\prime} H_{0}\right)^{k+1}\right]_{\mathbf{r r}}\right\}\right)
$$

The last result means that the first expression

$$
\operatorname{Tr}\left[H_{0}\left(\varphi \varphi^{\prime} H_{0}\right)^{N-1}\right]=\sum_{\mathbf{r}_{1}, \mathbf{r}_{2}, \ldots, \mathbf{r}_{N-1}} \operatorname{Tr}_{2}\left(H_{0 ; \mathbf{r}_{1} \mathbf{r}_{2}} \varphi_{\mathbf{r}_{2}} \varphi_{\mathbf{r}_{2}}^{\prime} H_{0 ; \mathbf{r}_{2} \mathbf{r}_{3}} \cdots \varphi_{\mathbf{r}_{N-1}} \varphi_{\mathbf{r}_{N-1}}^{\prime} H_{0 ; \mathbf{r}_{N-1} \mathbf{r}_{1}}\right)
$$


is nonzero only when $\mathbf{r}_{1}$ is different from all the other sites $\left\{\mathbf{r}_{j}\right\}(j=2, \ldots, N-1)$. This can be inserted in the Grassmann integral with the expression (B2) by borrowing a factor $-8 \epsilon \varphi_{\mathbf{r}_{1}} \varphi_{\mathbf{r}_{1}}^{\prime}$ from the first exponential factory to obtain Eq. (19).

For the product $\varphi_{\overline{\mathbf{r}}} \varphi_{\overline{\mathbf{r}}^{\prime}}^{\prime} \operatorname{det}\left(\mathbf{1}+\varphi \varphi^{\prime}-\varphi h \varphi^{\prime} h^{\dagger}\right)^{-1}$ we get

$$
\begin{gathered}
\varphi_{\overline{\mathbf{r}}} \varphi_{\overline{\mathbf{r}}^{\prime}}^{\prime} \exp \left[-8 \epsilon \sum_{\mathbf{r}} \varphi_{\mathbf{r}} \varphi_{\mathbf{r}}^{\prime}\right] \\
{\left[1-\frac{1}{\bar{\eta}} \sum_{l=0}^{N-1}\left\{\operatorname{Tr}\left[\left(\varphi \varphi^{\prime} A^{\prime}\right)^{l} \varphi \varphi^{\prime} H_{0}^{2}\right]-\sum_{k=0}^{l} \operatorname{Tr}\left[\left(\varphi \varphi^{\prime} A^{\prime}\right)^{l-k}\left(-\varphi A \varphi^{\prime}\right)^{k} \varphi H_{0} \varphi^{\prime} H_{0}\right]\right\}+O\left(\bar{\eta}^{-2}\right)\right]}
\end{gathered}
$$

and with $\mathbf{r} \neq \mathbf{r}^{\prime}$

$$
=\frac{i^{N-2}}{\bar{\eta}} \varphi_{\overline{\mathbf{r}}} \varphi_{\overline{\mathbf{r}}^{\prime}}^{\prime} \sum_{k=0}^{N-2} \operatorname{Tr}\left[\left(\varphi \varphi^{\prime} H_{0}\right)^{N-2-k}\left(-\varphi H_{0} \varphi^{\prime}\right)^{k} \varphi H_{0} \varphi^{\prime} H_{0}\right]+O\left(\bar{\eta}^{-2}\right) .
$$

Moreover, we have

$$
\operatorname{Tr}\left[\left(\varphi \varphi^{\prime} H_{0}\right)^{N-2-k}\left(-\varphi H_{0} \varphi^{\prime}\right)^{k} \varphi H_{0} \varphi^{\prime} H_{0}\right]=\operatorname{Tr}\left[\left(H_{0} \varphi \varphi^{\prime}\right)^{N-2-k} H_{0} \varphi\left(H_{0} \varphi \varphi^{\prime}\right)^{k} H_{0} \varphi^{\prime}\right],
$$

which also reads

$$
\begin{gathered}
\sum_{\mathbf{r}_{1}, \mathbf{r}_{2}, \ldots, \mathbf{r}_{N-1}} \operatorname{Tr}_{2}\left(\left[H_{0 ; \mathbf{r}_{1} \mathbf{r}_{2}} \varphi_{\mathbf{r}_{2}} \varphi_{\mathbf{r}_{2}}^{\prime} \cdots H_{0 ; \mathbf{r}_{N-3-k} \mathbf{r}_{N-2-k}} \varphi_{\mathbf{r}_{N-2-k}} \varphi_{\mathbf{r}_{N-2-k}}^{\prime}\right] H_{0 ; \mathbf{r}_{N-2-k} \mathbf{r}_{N-1-k}} \varphi_{\mathbf{r}_{N-1-k}}\right. \\
\left.\left[H_{0 ; \mathbf{r}_{N-1-k} \mathbf{r}_{N-k}} \varphi_{\mathbf{r}_{N-k}} \varphi_{\mathbf{r}_{N-k}}^{\prime} \cdots H_{0 ; \mathbf{r}_{N-1} \mathbf{r}_{N}} \varphi_{\mathbf{r}_{N}} \varphi_{\mathbf{r}_{N}}^{\prime}\right] H_{0 ; \mathbf{r}_{N} \mathbf{r}_{1}} \varphi_{\mathbf{r}_{1}}^{\prime}\right)
\end{gathered}
$$

When we multiply this expression with $\varphi_{\overline{\mathbf{r}}} \varphi_{\overline{\mathbf{r}}^{\prime}}^{\prime}$ it gives a nonzero result only if $\mathbf{r}_{N-1-k}=\overline{\mathbf{r}}^{\prime}$ and $\mathbf{r}_{1}=\overline{\mathbf{r}}$. Thus we get for the expression in (B5)

$$
-\frac{i^{N-2}}{\bar{\eta}} \sum_{k=0}^{N-2} \operatorname{Tr}_{2}\left[\left(H_{0} \varphi \varphi^{\prime}\right)_{\overline{\mathbf{r}} \overline{\mathbf{r}}^{\prime}}^{N-1-k}\left(H_{0} \varphi \varphi^{\prime}\right)_{\overline{\mathbf{r}}^{\prime} \mathbf{r}}^{k+1}\right]+O\left(\bar{\eta}^{-2}\right) .
$$

[1] F.D.M. Haldane and S. Raghu, Phys. Rev. Lett. 100, 013904 (2008).

[2] S. Raghu and F.D.M. Haldane, Phys. Rev. A 78, 033834 (2008).

[3] X. Cheng et al., Nature Mater. 15, 542-548 (2016).

[4] M.I. Mishchenko, L.D. Travis and A.A. Lacis, Multiple scattering of light by particles (Cambridge University Press 2006).

[5] K. Ziegler, J. Phys. A: Math. Theor. 50, 125002 (2017).

[6] K. Ziegler, Ann. Phys. 529 (8), 1600345 (2017).

[7] F. Wegner, Z. Phys. B 35, 207 (1979).

[8] L. Schäfer and F. Wegner, Z. Physik B 38, 113 (1980).

[9] K. Ziegler, Phys. Rev. B 55, 10661 (1997); Phys. Rev. Lett. 80, 3113 (1998).

[10] K. Ziegler, Phys. Rev. Lett. 102, 126802 (2009).

[11] K. Ziegler, Phys. Rev. B 79, 195424 (2009).

[12] K. Ziegler, J. Phys. A: Math. Theor. 48, 055102 (2015).

[13] R.P. Feynman and A.R. Hibbs, Quantum mechanics and Path Integrals (Dover Publications 2010).

[14] J. Glimm and A. Jaffe, Quantum Physics (Springer-Verlag 1981).

[15] L.S. Schulman, Techniques and Applications of Path Integration (Dover Publications 2005).

[16] F.A. Berezin, The method of second quantization (Academic Press 1966).

[17] J.W. Negele and H. Orland, Quantum Many-Particle Physics (Addison-Wesley 1988).

[18] K. Ziegler, J. Phys. A: Math. Theor. 45, 335001 (2012). 
[19] C. Itzykson and J.-M. Drouffe, Statistical Field Theory Volume 1 (Cambridge University Press 1989).

[20] B.M. McCoy and T.T. Wu, The two-dimensional Ising model (Harvard University Press 1973).

[21] F.J. Dyson, Phys. Rev. 92, 1331 (1953).

[22] G. Theodorou and M.H. Cohen, Phys. Rev. B 13, 4597 (1976).

[23] T.P. Eggarter and R. Riedinger, Phys. Rev. B 18, 569 (1978).

[24] C.M. Soukoulis, I. Webman, G.S. Grest, and E.N. Economou, Phys. Rev. B 26, 1838 (1982).

[25] M. Inui, S.A. Trugman, and E. Abrahams, Phys. Rev. B 49, 3190 (1994).

[26] J.T Chalker and P.D Coddington, Journal of Physics C: Solid State Physics 21, 2665 (1988).

[27] J.T. Chalker, N. Read, V. Kagalovsky, B. Horovitz, Y. Avishai, A.W.W. Ludwig, Phys. Rev. B 65012506 (2001).

[28] Y. Kang et al., Nat. Commun. 93029 (2018). 